\title{
Species Diversity of Candida from Hospital Environment in Plateau State, Nigeria
}

\author{
Nnaemeka Emmanuel Nnadi ${ }^{1}$, Miri Alexander Nanpyal ${ }^{1}$, Nanpon Kaduna ${ }^{1}$, Bege Jonathan ${ }^{2}$, \\ Iskender Karalti ${ }^{3,4}$ \\ ${ }^{1}$ Department of Microbiology, Faculty of Natural and Applied Sciences, Plateau State University, Bokkos, Nigeria \\ ${ }^{2}$ Environmental \& Occupational Toxicology Unit, Department of Biochemistry, Faculty of Natural and Applied Sciences, Plateau State \\ University, Bokkos, Nigeria \\ ${ }^{3}$ Azerbaijan Medical University, Educational and Therapeutic Hospital, Central Laboratory, Baku, Azerbaijan \\ ${ }^{4}$ Yeditepe University, Faculty and Health Sciences, Department of Nutrition and Dietetics, İstanbul, Turkey
}

\section{Email address:}

eennadi@gmail.com (N. E. Nnadi), mirinanpyal@gmail.com (M. A. Nanpyal), nanponkaduna@gmail.com (N. Kaduna), begejonathan@plasu.edu.ng (B. Jonathan), Iskender.karalti@gmail.com (I. Karalti)

${ }^{*}$ Corresponding author

\section{To cite this article:}

Nnaemeka Emmanuel Nnadi, Miri Alexander Nanpyal, Nanpon Kaduna, Bege Jonathan, Iskender Karalti. Species Diversity of Candida from Hospital Environment in Plateau State, Nigeria. American Journal of Biomedical and Life Sciences. Vol. 8, No. 1, 2020, pp. 15-19. doi: 10.11648 j.ajbls.20200801.14

Received: January 8, 2020; Accepted: January 20, 2020; Published: February 7, 2020

\begin{abstract}
In the past few years the role the hospital environment in the transmission of infections has increased leading to the increase in the incidence of morbidity and mortality associated with Candida infections. The hospital environment can serve as an alternative niche for the presence of opportunistic pathogens of public health concern. This study sought to use molecular approach to study the prevalence of Candida from hospital environments in Jos, Plateau State, Nigeria. Samples were collected from different hospital environments and cultured on Chromagar Candida media. Taqman PCR was then used to characterize the isolates, the ability of Candida to form biofilm was evaluated. In this study, Candida parapsilosis with $31.25 \%$ was the most prevalent Candida followed by Candida (Nakaseomyces) glabrata and Candida krusei with prevalence of $28.13 \%$. The ability to form biofilms was evaluated for these environmental isolates, and it was observed that $C$. parapsilosis biofilm formation correlated with high prevalence with $C$. parapsilosis having high biofilm formation (20/64) followed by C. glabrata and C. krusei (18/64) each Environmental sampling has the potential to improve our knowledge about yeast biodiversity and the evolution of pathogenic yeasts with clinical relevance. This study is the first in Nigeria to study to characterize Candida from hospital environments in Jos, Plateau state Nigeria.
\end{abstract}

Keywords: Hospital Environments, Candida, Taqman PCR, Biofilm

\section{Introduction}

Fungal nosocomial infections have increased in frequency in the past few years [1] and the Hospital environment has played a major role in the transmission of nosocomial Candida infections [2, 3]. The frequency of hospital acquired fungal infections have increased in recent times with concomitant increases in morbidity and mortality. The recent outbreak of multi-resistant strain of C. auris [4] a pathogen with no known ecology but has been isolated from hospital environment that has been an increase need to look at our hospital environment to find out how safe they are for patients and visitors. There is a shift away from Candida albicans as a major causes of candidemia to non-albicans Candida (NAC). Cases of NAC reported from hospital environment include C. auris [3, 5, 6], C. parapsilosis [2, 7], C. glabrata [8]. In a recent study by Opulente et al. [9] they showed that these common pathogenic yeast have ecological niches outside the clinical settings and may persist in alternative niches with ecology that may be more 
complicated than is currently assumed. There is a dogma that the infective strains of the human normal gut flora $(C$. albicans and C. glabrata, and the skin for C. parapsilosis) comes from the patients endogenous flora, however the isolation of these pathogens from alternative niches tends to challenge this dogma [10]

The hospital environment harbor a lot of other pathogens, these pathogens may be transmitted to individuals in many ways ranging from hands of healthcare providers [7], catheters [11] and contact with hospital environments like beds [12]. In Nigeria, little or nothing is known about the epidemiology of fungal infections and the species distribution of Candida pathogens in the hospital environment. This study sought to use molecular approach to characterize isolates of Candida from hospital environment in Plateau State, Nigeria.

\section{Materials and Methods}

Yeast samples were obtained from different hospital environments (laboratory, wards, doors handle, bench surfaces and hands of health workers and air) sources in a secondary care hospital, and only those that contained yeasts were maintained for further processing. Samples were collected from hospital environments, that is, seats used patients visiting the hospital doors handle, sheets. Environmental air samples were collected in 4-cm diameter petri dishes containing Sabouraud agar, which were exposed to the environment for $25 \mathrm{~min}$.

Samples from surfaces were collected using sterile swabs sticks and were transported to the Microbiology Laboratory, Department of Microbiology of Plateau State University Bokkos within an hour after collection. Each swab was rolled onto Sabouraud dextrose agar (SDA) plate supplemented with chloramphenical $(0.5 \mathrm{~g} / \mathrm{L})$ and incubated at $37^{\circ} \mathrm{C}$ for 2 days.

Distinct yeast colonies from each culture plate were subcultured on HiCrome Candida differential media plates for the identification of Candida species. These plates were incubated at $37^{\circ} \mathrm{C}$ for 2 days. Identification was done by the specific colour of colonies on the media as described by the manufacturers. Subcultures were made from chromogenic agar plates onto Sabouraud dextrose agar slants and sterile water. Slants were examined for growth after 24-48 hours incubation at $37^{\circ} \mathrm{C}$ and stored. These slants served as stocks from which subcultures were made for further characterization of the isolates.

\subsection{Identification of the Candida Species Using Taqman Probes}

MagNA Pure Compact Nucleic Acid Isolation Kit I and MagNA Pure Compact (Roche, Germany) was used for nucleic acid isolation. Briefly, Colonies were obtained from pure culture on a Petri dish and put in to an eppendorf tube. Then $500 \mu l$ lyticase lysis buffer ( $50 \mathrm{mM}$ Tris (pH: 7.6), $1 \mathrm{mM}$
EDTA (pH: 8.0), \%2 2-merkaptoetanol, 10 U lyticase (Sigma) were added and incubated at $37^{\circ} \mathrm{C}$ for one hour. Five hundred-(500) $\mu \mathrm{l}$ supernatant was taken and put in to automatic nucleic acid isolation machine. The final volume was adjusted for $100 \mu$ l. Taqman probes (Table 1) were used for identification of the Candida species.

PCR Conditions. Light Cycler 2.0 (Roche, Germany) and Light Cycler Taqman Master (Roche, Germany) was used for real time PCR. It was used this PCR conditions: Initial denaturation is $10 \mathrm{~min}$ at $95^{\circ} \mathrm{C}$; Amplification was performed 45 cycle (denaturation is $10 \mathrm{sec}$. at $95^{\circ} \mathrm{C}$; annealing is $20 \mathrm{sec}$ at $53^{\circ} \mathrm{C}$; extension is $1 \mathrm{sec}$ at $72^{\circ} \mathrm{C}$ (single)) and followed by cooling step was $30 \mathrm{sec}$ at $40^{\circ} \mathrm{C}$. Finally analyzes were performed by Light Cycler program. VITEK was used to confirm the presence of $C$. famata.

\subsection{Biofilm Production}

Biofilm production was detected by tube method described by Branchini et al (1994) [13], and evaluated using the method of Shin et at [14]. A loopful of organisms from sabouraud's Dextrose agar (SDA) plate was inoculated into Sabouraud's Dextrose Broth supplemented with glucose (final concentration $8 \%$ ). Polystyrene microtiter plates were then incubated at $37^{\circ} \mathrm{C}$ for 24 hours after which the broth was aspirated out gently. The microtiter wells were then washed once with distilled water and then stained with $1 \%$ safranin. The microtiter plate was kept still for 7 minutes. Safranin was then removed, and the wells were examined for biofilm production. Biofilm production was read independently by two different observers. The adherent biofilm layer was scored visually as either negative or weak positive $(+)$, or moderate positive $(++)$ or strong positive $(+++)$. All positive results, including weak or strong, were considered as positive.

\section{Results and Discussion}

There has been a recent increase in NAC associated infection in hospitalized patients [15]. These species have also shown reduced susceptibility to some antifungals and have posed a problem to clinicians. In this study, a high prevalence was observed for $C$. parapsilosis $31.25 \%$ followed by $C$. glabarata and $C$. krusei with a prevalence of $28.13 \%$ each (Table 1). The high prevalence of $C$. parapsilosis is consistent with most studies that have shown a high occurrence of this species in hospital environment and in association with Catheter tips [16]. C. parapsilosis accounts for a significant proportion of nosocomial infections with an increasing prevalence in hospital settings [2, 17]. It has been reported as a major cause of candidemia in paediatric units [18] with exogenous and environmental sources identified as the source of infection [2]. This study contrasts what was reported by Savastano el al. (2016) [8] in Brazil, in their study, they found a high occurrence of $C$. albicans followed by C. glabrata and C. parapsilosis in that order. 
Table 1. Taqman probes which used for identification of Candida species.

\begin{tabular}{ll}
\hline Species Name & Tagman probes \\
\hline Candida albicans & FAM-CATTGCTTGCGGCGGTA-TAMRA $(0.2 \mathrm{M})$ \\
Candida tropicalis & FAM-GGCCACCACAATTTATTTCA-TAMRA $(0.2 \mathrm{M})$ \\
Candida parapsilosis & FAM-GAAAGGCGGAGTATAAAC-TAMRA $(0.2 \mathrm{M})$ \\
Candida glabrata & FAM-GTTTTACCAACTCGGTGTTGAT-TAMRA $(0.2 \mathrm{M})$ \\
\hline
\end{tabular}

Different distribution have been reported through out the world, C. albicans and C. glabrata were the most frequently isolates from Denmark and the USA [19]. The distribution of the Candida isolates have changed in the last decased with the decrease in the proportion of $C$. albicans and with and increase in C. paraspsilosis and C. glabrata infections [20]. An increasing trend have been observed for candidemias caused by non-albicans Candida, in the US, C. glabrata accounts for one-third of the candidemias [21] followed by $C$. parapsilosis which accounts for $15 \%$ of all isolates [22]. In South Africa, C. albicans and C. parapsilosis are the dominant species found [23]. In Nigeria however, the diversity of Candida species implicated in candidemia is not known as well as the distribution of Candida genotypes from hospital environment is scarce. Our study is the first to look at occurrence of Candida species in hospital environment in Nigeria. According to our results, C. tropicalis and C. famata had the lowest rate of isolation, as also reported in another study [24]. Although a different isolation frequency has been reported for C. tropicalis in another study. Hallur, Mahapatra, Deb, Tripathy, Misra and Praharaj [25] reported a higher occurrence of $C$. tropicalis. C. famata (Debaryomyces hansenii) yeast commonly found in natural substrates and in various types of cheese [26], although it has been reported in human infections its occurrence is rare [27, 28]. It has been misidentified as Pichia guilliermondii, P. caribbica, P. jadinii, D. hansenii, Candida palmioleophila, C. haemulonii type II, and Clavispora lusitaniae. Suggesting therefore that $C$. famata may not be a human pathogen as thought [29].

Table 2. Distribution of Candida species isolated from Hospital environments in Plateau state, Nigeria.

\begin{tabular}{lllll}
\hline Candida species & Catheter tips & Hospital (AIR) & Other Surfaces & Total \\
\hline Candida parapsilosis & 20 & 0.0 & 0.0 & 2 \\
Candida tropicalis & 4 & 1 & 10 & $5(6.25)$ \\
Candida krusei & 0.0 & 8 & 12 & $18(28.13)$ \\
Candida glabrata & 0.0 & 6 & 0.0 & $18(28.13)$ \\
Candida famata & 0.0 & 3 & 24 & $3(4.69)$ \\
Total & 4 & 18 & 64 & \\
\hline
\end{tabular}

The adherence capacity of diverse Candida species play a role in colonization and subsequent invasion of these Candida species as well as the perpetuation of infection caused by these various species [30]. Several factors can predispose a person to Candida colonization, these factors include gastrointestinal tract colonization by Candida species resulting from prolonged use of broad-spectrum antibacterial agents, disruption of the gastrointestinal mucosal surfaces by cytotoxic agents or hypotension, and neutropenia [31]. Central venous catheters (CVCs), however, appear to be the most common risk factor for the development of candidemia in patients without neutropenia or major immunodeficiencies [32]. Biofilms formation on prosthetic materials have been associated with enhanced virulence of $C$. parapsislosis [13]. All the Candida isolated obtained in this study produced biofilms. The rank order of biofilm production of these Candida isolates is shown in table 3. Considerable difference exist in the way different species formed biofilms.

Table 3. Biofilm Production by some of the Candida isolates obtained in this study.

\begin{tabular}{|c|c|c|c|c|c|}
\hline Candida species & Strong Positive $(+++)$ & Moderate Positive (++) & Weak Positive $(+)$ & Weak or Negative (0/-) & Total \\
\hline Candida parapsilosis & 1 & 0.0 & 8 & 11 & 20 \\
\hline Candida tropicalis & 0 & 1 & 1 & 3 & 5 \\
\hline Candida krusei & 0.0 & 3 & 7 & 8 & 18 \\
\hline Candida glabrata & 2 & 4 & 12 & 3 & 18 \\
\hline Candida famata & 0.0 & 0.0 & 0.0 & 3 & 3 \\
\hline Total & 3 & 8 & 28 & 28 & 64 \\
\hline
\end{tabular}

\section{Conclusion}

This study has reported for the first time the species diversity of Candida isolates that are found in a hospital environment in Nigeria. This paper provides a baseline, whereby future studies have the potential to demonstrate a rise in non-albicans species and their possible role in pathogenesis. These would go a long way in stimulating research to finding out which species of Candida is more 
prevalent in patients with candidemia in Nigeria and would help clinicians in the management of such cases.

\section{Acknowledgements}

N. N. E received support from the Tertiary Education Trust Fund (TetFund) through its Institutional Based Research (IBR) intervention at Plateau State University, Bokkos, Nigeria.

\section{References}

[1] U. Binder, C. Lass-Flörl, Epidemiology of invasive fungal infections in the mediterranean area, Mediterranean journal of hematology and infectious diseases, 3 (2011).

[2] R. Sabino, P. Sampaio, L. Rosado, Z. Videira, F. Grenouillet, C. Pais, Analysis of clinical and environmental Candida parapsilosis isolates by microsatellite genotyping - a tool for hospital infection surveillance, Clinical Microbiology and Infection, 21 (2015) 954. e951-954. e958.

[3] S. Schelenz, F. Hagen, J. L. Rhodes, A. Abdolrasouli, A. Chowdhary, A. Hall, L. Ryan, J. Shackleton, R. Trimlett, J. F. Meis, First hospital outbreak of the globally emerging Candida auris in a European hospital, Antimicrobial Resistance \& Infection Control, 5 (2016) 35.

[4] A. Casadevall, D. P. Kontoyiannis, V. Robert, On the emergence of Candida auris: climate change, azoles, swamps and birds, bioRxiv, (2019) 657635 .

[5] S. Vallabhaneni, Investigation of the first seven reported cases of Candida auris, a globally emerging invasive, multidrug-resistant fungus-United States, May 2013August 2016, MMWR. Morbidity and mortality weekly report, 65 (2016).

[6] S. R. Lockhart, K. A. Etienne, S. Vallabhaneni, J. Farooqi, A. Chowdhary, N. P. Govender, A. L. Colombo, B. Calvo, C. A. Cuomo, C. A. Desjardins, E. L. Berkow, M. Castanheira, R. E. Magobo, K. Jabeen, R. J. Asghar, J. F. Meis, B. Jackson, T. Chiller, A. P. Litvintseva, Simultaneous Emergence of Multidrug-Resistant Candida auris on 3 Continents Confirmed by Whole-Genome Sequencing and Epidemiological Analyses, Clin Infect Dis, 64 (2017) 134-140.

[7] D. Delfino, F. Scordino, I. Pernice, C. L. Passo, R. Galbo, A. David, I. Barberi, G. Criseo, A. Cascio, O. Romeo, Potential association of specific Candida parapsilosis genotypes, bloodstream infections and colonization of health workers' hands, Clinical Microbiology and Infection, 20 (2014) O946O951.

[8] C. Savastano, E. de Oliveira Silva, L. L. Gonçalves, J. M. Nery, N. C. Silva, A. L. T. Dias, Candida glabrata among Candida spp. from environmental health practitioners of a Brazilian Hospital, brazilian journal of microbiology, 47 (2016) 367-372.

[9] D. A. Opulente, Q. K. Langdon, K. V. Buh, M. A. Haase, K. Sylvester, R. V. Moriarty, M. Jarzyna, S. L. Considine, R. M. Schneider, C. T. Hittinger, Pathogenic budding yeasts isolated outside of clinical settings, FEMS yeast research, 19 (2019).

[10] F. Morio, Dear medical mycologists, it is time to look outside the box, FEMS yeast research, (2019).
[11] L. J. Douglas, Candida biofilms and their role in infection, Trends in microbiology, 11 (2003) 30-36.

[12] M. S. Rangel-Frausto, T. Wiblin, H. M. Blumberg, L. Saiman, J. Patterson, M. Rinaldi, M. Pfaller, J. E. Edwards Jr, W. Jarvis, J. Dawson, National epidemiology of mycoses survey (NEMIS): variations in rates of bloodstream infections due to Candida species in seven surgical intensive care units and six neonatal intensive care units, Clinical Infectious Diseases, 29 (1999) 253-258.

[13] M. Branchini, M. Pfaller, J. Rhine-Chalberg, T. Frempong, H. Isenberg, Genotypic variation and slime production among blood and catheter isolates of Candida parapsilosis, Journal of clinical microbiology, 32 (1994) 452-456.

[14] J. H. Shin, S. J. Kee, M. G. Shin, S. H. Kim, D. H. Shin, S. K. Lee, S. P. Suh, D. W. Ryang, Biofilm production by isolates of Candida species recovered from nonneutropenic patients: comparison of bloodstream isolates with isolates from other sources, Journal of Clinical Microbiology, 40 (2002) 12441248 .

[15] H. Badali, S. Rezaie, J. F. Meis, S. A. K. Afshari, M. Modiri, F. Hagen, M. Moazeni, R. Mohammadi, S. Khodavaisy, Microsatellite genotyping of clinical Candida parapsilosis isolates, Current medical mycology, 3 (2017) 15.

[16] R. A. Cordeiro, R. S. Brilhante, L. D. Pantoja, R. E. Moreira Filho, P. R. Vieira, M. F. Rocha, A. J. Monteiro, J. J. Sidrim, Isolation of pathogenic yeasts in the air from hospital environments in the city of Fortaleza, northeast Brazil, The Brazilian Journal of Infectious Diseases, 14 (2010) 30-34.

[17] L. R. Storti, G. Pasquale, R. Scomparim, A. L. Galastri, F. Alterthum, W. Gambale, C. R. Paula, Candida spp. isolated from inpatients, the environment, and health practitioners in the Pediatric Unit at the Universitary Hospital of the Jundiai Medical College, State of São Paulo, Brazil, Revista da Sociedade Brasileira de Medicina Tropical, 45 (2012) 225231.

[18] O. Romeo, D. Delfino, A. Cascio, C. L. Passo, M. Amorini, D. Romeo, I. Pernice, Microsatellite-based genotyping of Candida parapsilosis sensu stricto isolates reveals dominance and persistence of a particular epidemiological clone among neonatal intensive care unit patients, Infection, Genetics and Evolution, 13 (2013) 105-108.

[19] M. Pfaller, D. Diekema, Epidemiology of invasive candidiasis: a persistent public health problem, Clinical microbiology reviews, 20 (2007) 133-163.

[20] F. Lamoth, S. R. Lockhart, E. L. Berkow, T. Calandra, Changes in the epidemiological landscape of invasive candidiasis, Journal of Antimicrobial Chemotherapy, 73 (2018) i4-i13.

[21] E. Matsumoto, L. Boyken, S. Tendolkar, J. McDanel, M. Castanheira, M. Pfaller, D. Diekema, Candidemia surveillance in Iowa: emergence of echinocandin resistance, Diagnostic microbiology and infectious disease, 79 (2014) 205-208.

[22] A. A. Cleveland, L. H. Harrison, M. M. Farley, R. Hollick, B. Stein, T. M. Chiller, S. R. Lockhart, B. J. Park, Declining incidence of candidemia and the shifting epidemiology of Candida resistance in two US metropolitan areas, 2008-2013: results from population-based surveillance, PLoS One, 10 (2015) e0120452. 
[23] N. P. Govender, J. Patel, R. E. Magobo, S. Naicker, J. Wadula, A. Whitelaw, Y. Coovadia, R. Kularatne, C. Govind, S. R. Lockhart, Emergence of azole-resistant Candida parapsilosis causing bloodstream infection: results from laboratory-based sentinel surveillance in South Africa, Journal of Antimicrobial Chemotherapy, 71 (2016) 1994-2004.

[24] P. Pronovost, D. Needham, S. Berenholtz, D. Sinopoli, H. Chu, S. Cosgrove, B. Sexton, R. Hyzy, R. Welsh, G. Roth, An intervention to decrease catheter-related bloodstream infections in the ICU, New England Journal of Medicine, 355 (2006) 2725-2732.

[25] V. Hallur, A. Mahapatra, B. Deb, S. Tripathy, S. Misra, A. Praharaj, Comparison of virulence profile of C. albicans and C. tropicalis isolated from patients with Candida infection and hospital environment, MEDICAL MYCOLOGY, OXFORD UNIV PRESS GREAT CLARENDON ST, OXFORD OX2 6DP, ENGLAND, 2018, pp. S152-S152.

[26] M. Quirós, P. Martorell, M.-J. Valderrama, A. Querol, J. M. Peinado, M.-I. de Silóniz, PCR-RFLP analysis of the IGS region of rDNA: a useful tool for the practical discrimination between species of the genus Debaryomyces, Antonie van Leeuwenhoek, 90 (2006) 211-219.

[27] D. Wagner, A. Sander, H. Bertz, J. Finke, W. Kern, Breakthrough invasive infection due to Debaryomyces hansenii (teleomorph Candida famata) and Scopulariopsis brevicaulis in a stem cell transplant patient receiving liposomal amphotericin B and caspofungin for suspected aspergillosis, Infection, 33 (2005) 397-400.
[28] D. Diekema, S. Messer, A. Brueggemann, S. Coffman, G. Doern, L. Herwaldt, M. Pfaller, Epidemiology of candidemia: 3-year results from the emerging infections and the epidemiology of Iowa organisms study, Journal of Clinical Microbiology, 40 (2002) 1298-1302.

[29] M. Desnos-Ollivier, M. Ragon, V. Robert, D. Raoux, J.-C. Gantier, F. Dromer, Debaryomyces hansenii (Candida famata), a rare human fungal pathogen often misidentified as Pichia guilliermondii (Candida guilliermondii), Journal of clinical microbiology, 46 (2008) 3237-3242.

[30] B. Almirante, D. Rodríguez, B. J. Park, M. Cuenca-Estrella, A. M. Planes, M. Almela, J. Mensa, F. Sanchez, J. Ayats, M. Gimenez, Epidemiology and predictors of mortality in cases of Candida bloodstream infection: results from populationbased surveillance, Barcelona, Spain, from 2002 to 2003, Journal of Clinical Microbiology, 43 (2005) 1829-1835.

[31] G. T. Cole, A. A. Halawa, E. J. Anaissie, The role of the gastrointestinal tract in hematogenous candidiasis: from the laboratory to the bedside, Clinical Infectious Diseases, 22 (1996) S73-S88.

[32] J. H. Rex, J. E. Bennett, A. M. Sugar, P. G. Pappas, C. M. Van Der Horst, J. E. Edwards, R. G. Washburn, W. M. Scheld, A. W. Karchmer, A. P. Dine, A randomized trial comparing fluconazole with amphotericin B for the treatment of candidemia in patients without neutropenia, New England Journal of Medicine, 331 (1994) 1325-1330. 